\title{
Formação de professores de matemática para o uso efetivo de tecnologias em sala de aula
}

\author{
Vandoir Stormowski - PUCRS - vandoir@yahoo.com.br \\ Maria Alice Gravina - UFRGS - gravina@mat.ufrgs.br \\ José Valdeni de Lima - UFRGS - valdeni@inf.ufrgs.br
}

\begin{abstract}
Resumo: apesar do desenvolvimento das tecnologias digitais em diversas áreas, estudos apontam que estes recursos ainda são utilizados de forma insuficiente ou precária no processo de ensino e aprendizagem de matemática. Neste cenário, o artigo apresenta um estudo em andamento com o objetivo de qualificar a apropriação da tecnologia por parte dos professores de matemática. O estudo se fundamenta na teoria da abordagem instrumental (Rabardel) no que se refere ao processo de apropriação de um artefato (software GeoGebra) e na teoria dos registros de representação (Duval) no que diz respeito à articulação do conhecimento matemático através do uso de representações dinâmicas. A partir deste enfoque, são apresentadas estratégias de formação que possam qualificar o processo de apropriação da tecnologia pelos professores de matemática.
\end{abstract}

Palavras-chave: tecnologias digitais; representações dinâmicas; professores de matemática.

\section{Training of mathematics teachers for the effective use of technology in the classroom}

\begin{abstract}
: despite the development of digital technologies in various fields, studies show that these resources are still used insufficiently or precariously in education and mathematics learning process. In this scenario, the article presents a study in progress in order to qualify the appropriation of technology by math teachers. The study is based on the theory of instrumental approach (Rabardel) with regard to the ownership of an artifact process (GeoGebra software) and the theory of representation registers (Duval) in respect of the combination of mathematical knowledge through the use of dynamic representations. From this approach, training strategies are presented that might qualify the process of appropriation of technology by mathematics teachers.
\end{abstract}

Keywords: digital technologies; dynamic representations; math teachers.

\section{Introdução}

O desenvolvimento tecnológico avança em todas as áreas, e possui contribuições significativas na área de educação, mas ainda há muito a avançar. Para identificar as contribuições na área de educação, basta observar a quantidade de programas de pósgraduação existentes, que abordam, em essência, o uso de tecnologias digitais na educação. Tanto a produção dos pesquisadores destes programas, quanto a diversidade de revistas nacionais e internacionais com este enfoque, confirmam as contribuições e potencialidades do uso da tecnologia no processo de aprendizagem escolar.

Em contrapartida, pesquisas (BARBOSA, 2012; 2014) apontam que a contribuição destes estudos, e a potencialidade dos recursos digitais, ainda não chegou de forma efetiva e substancial no contexto escolar. 
Mesmo em outros países do mundo, estudos que abordam o uso de tecnologias digitais no ensino de matemática, tal como Artigue (2010) e Joubert (2013), destacam que a tecnologia ainda é pouco utilizada em sala de aula, e que que o potencial já identificado destas tecnologias continua sendo pouco explorado. Os autores afirmam ainda, que as pesquisas na área não estão conseguindo contribuir tanto quanto se esperava para a inserção das tecnologias no contex to escolar.

Na busca de respostas para esta situação, Artigue (2010) afirma que é consenso que a evolução dos sistemas educacionais depende do desenvolvimento profissional dos professores e da qualidade de sua formação. Ou seja, é importante que as escolas sejam providas de recursos digitais, mas é fundamental e essencial a qualificação da formação de professores, para que de fato as potencialidades contribuam para a aprendizagem dos alunos.

Em pesquisa realizada pelo realizada pelo Comitê Gestor da Internet no Brasil (CGI.br) envolvendo alunos, professores e coordenadores de escolas públicas e privadas de áreas urbanas de todo o Brasil, constatou-se que cerca de metade dos professores já realizou curso específico para capacitação no uso de recursos tecnológicos, e mesmo assim, dois terços dos professores consideram que os alunos dominam melhor estes recursos do que eles próprios (BARBOSA, 2014). E mesmo com investimentos na capacitação de professores, os dados mostram que os resultados ainda são pouco efetivos (BARBOSA, 2012, p. 30), apontando que a sala de aula ainda se caracteriza essencialmente por aula expositiva, exercícios de fixação e interpretação de texto.

Desta forma, considerando que a formação dos professores para o uso de tecnologias é essencial, mas ao mesmo tempo deficiente em sua execução, estamos desenvolvendo um estudo com o objetivo de qualificar a formação dos professores de matemática. O foco do estudo está na elaboração de um processo formativo que explore as potencialidades dos recursos digitais, e que possibilite os professores participantes articular a tecnologia em contexto escolar de forma mais efetiva, contribuindo efetivamente para o processo de aprendizagem de seus alunos.

Há de se salientar também que grande parte dos professores atuantes nas escolas, possui pouca flexibilidade de horários para participar de cursos de formação, e nem sempre há disponibilidade de tempo para se deslocar para as cidades com centros de formação. Desta forma, considerando que mais de $90 \%$ dos professores acessam a internet regularmente (BARBOSA, 2014), vislumbramos nos cursos na modalidade EAD uma contribuição efetiva para concretizar esta formação necessária.

A partir deste cenário, este artigo apresenta o aporte teórico utilizado neste estudo em desenvolvimento, e as respectivas implicações na estruturação do curso de formação de professores de matemática para apropriação da tecnologia. Para ilustrar estas contribuições, serão apresentados exemplos de atividades que articulam o uso da tecnologia com o processo de aprendizagem em matemática. Os exemplos apresentados ilustram o uso do software GeoGebra, mas as implicações são válidas para qualquer outro recurso educacional com foco na aprendizagem de matemática.

A seção 2 é destinada ao processo de aprendizagem matemática, com atenção aos registros de representação semiótica (Duval, 2003; 2009) e ás características do conhecimento matemático mobilizado através de recursos digitais. $\mathrm{Na}$ seção 3 é apresentado brevemente o aporte teórico utilizado para entender o processo de apropriação da tecnologia pelos professores (RABARDEL, 1995). A seção 4 traz algumas implicações deste estudo na elaboração do processo de formação, com a apresentação de 
alguns exemplos de atividades desenvolvidas, e apontamentos na direção de resultados deste estudo em desenvolvimento.

\section{Representações semióticas}

Antes de entender o processo de apropriação de um software, tal como o GeoGebra, é preciso perceber a importância a ser dada aos sistemas de signos e suas regras no ensino e aprendizagem da matemática, tal como apontam diversas pesquisas que possuem na Teoria dos Registros de Representação Semiótica (DUVAL, 2003; 2009) uma fonte importante.

A Matemática é, por excelência, uma área de conhecimento que depende de sistemas de representação que fazem uso da linguagem natural e de signos com regras de uso bem estabelecidas, que são os sistemas de representação semiótica. Ernest (2006), apresenta uma sistematização, e os organiza em três componentes: um conjunto de signos expressos através da escrita (aqui incluindo a linguagem natural) e do desenho; um conjunto de regras de produção e de organização dos signos que vão dar sentido ao discurso; um conjunto de relações entre signos e seus significados, que dependem da estrutura matemática subjacente que está sendo considerada.

Duval utiliza o termo registro para designar diferentes tipos de representações e, em termos amplos, classifica em quatro tipos: língua natural, sistemas de escritas (numérica, algébrica e simbólica), figuras geométricas e gráficos (2003, p. 14).

Para Duval (2006), objeto matemático e sua representação são distintos, e as diferentes representações de um objeto trazem entendimentos parciais sobre o mesmo. Por exemplo, a representação gráfica de uma função tem características que são diferentes daquelas que se tem na representação algébrica da mesma, e as possibilidades de entendimento do objeto matemático 'função', nos diferentes registros, também são diferentes. A atividade matemática, na sua essência, faz uso simultâneo de mais de um registro de representação, para um mesmo objeto. Esta possibilidade de trânsito entre diferentes registros ajuda no entendimento do objeto matemático e este é um dos aspectos que diferencia a matemática de outras áreas de estudo: "ao contrário de outras áreas de conhecimento, os signos e a transformação das representações semióticas são centrais na atividade matemática" (DUVAL, 2006, p. 107).

Para um melhor entendimento do funcionamento dos registros de representação, Duval introduz o conceito de transformação de registros e os classifica em dois tipos:

- tratamento: é uma transformação de uma representação semiótica em outra representação semiótica, dentro de um mesmo registro.

- conversão: é uma transformação de uma representação semiótica em outra representação semiótica, dentro de outro registro.

A conversão é a transformação que, primordialmente, conduz à compreensão matemática, e por isso deve ser colocada sob atenção especial no ensino da matemática (DUVAL, 2003, p.16). É uma maior complexidade cognitiva que se tem na conversão, comparada com aquela que se tem no tratamento. Isto pode ser explicado, através do fenômeno de não-congruência entre representações: isto acontece quando a correspondência entre representação inicial com a representação obtida após a transformação não é totalmente explícita. 
Segundo Duval (2009), não há aprendizagem matemática sem a utilização de sistemas de signos, e seu processo passa essencialmente pelo tratamento e conversão de registros, sendo este último o mais importante.

E é este conhecimento matemático, representado por sistemas de signos, que está articulado nos softwares de Matemática Dinâmica (MD). Estes softwares, tal como o GeoGebra, permitem a 'manipulação dinâmica' de conhecimento de várias áreas da matemática, tal como geometria, álgebra, funções, estatística, etc.

Os softwares de MD articulam o conhecimento matemático destes diferentes registros (língua natural, geométrico, gráfico e sistema de numeração) com algumas particularidades, que passaremos a comentar.

A primeira particularidade está no dinamismo das representações, que se caracteriza pela possibilidade de mover elementos matemáticos representados, sem que estes percam as propriedades subjacentes (propriedades oriundas de sua representação no software). Na verdade, é movimentando os pontos e elementos representados (dinamismo), que as propriedades podem ser observadas, mesmo quando não são muito evidentes.
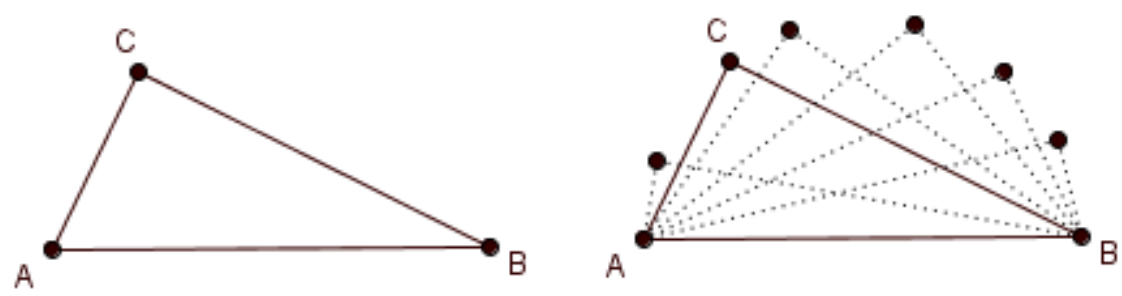

Figura 1: triângulo representado no GeoGebra

$\mathrm{Na}$ figura 1, temos à esquerda a representação de um triângulo $\mathrm{ABC}$ no GeoGebra. Este triângulo, à primeira vista, dá a impressão de ser um triângulo qualquer. Mas, movimentando o vértice $C$, as diferentes posições que o mesmo pode apresentar (na figura à direita, os triângulos pontilhados indicam algumas das diferentes posições que o vértice $\mathrm{C}$ pode ocupar), indicam que este vértice se movimenta sobre uma semicircunferência. Ou seja, a limitação de movimento do vértice $\mathrm{C}$, confere ao triângulo uma propriedade particular, a saber: $\mathrm{ABC}$ é triângulo retângulo em C. Esta propriedade está implícita, e nem sempre possui identificação fácil, pois decorre de um teorema da geometria euclidiana.

Este tipo de exploração de propriedades, em que se articula a representação com conhecimento matemático subjacente, é muito importante para o processo de formação de professores de matemática para o uso de tecnologia. É nela que se encontra o potencial de exploração e simulação, que transforma o software em ambiente rico para o desenvolvimento de atividades didáticas.

É possível observar que no lado direito da figura 1, há o processo de tratamento no registro geométrico, pois as diferentes posições do ponto $\mathrm{C}$ apresentam diferentes representações do mesmo triângulo $\mathrm{ABC}$, e sempre utilizando representação geométrica. Segundo Duval (2009), este é um dos processos importantes para a aprendizagem matemática.

O tratamento também pode ser observado na figura 2, em que o gráfico da função $f(x)=x^{2}$, é deslocado horizontalmente. O gráfico em forma de parábola apresenta exatamente o mesmo formato, mas em diferentes posições no plano cartesiano. $\mathrm{Na}$ 
correspondência entre o gráfico (à direita) e sua expressão (algébrica), temos o processo de conversão de registros. Ou seja, são diferentes representações (gráfico e representação algébrica) do mesmo elemento matemático. As diferentes posições do gráfico, estão relacionadas a diferentes expressões algébricas. E aqui um adendo importante: o deslocamento horizontal é de difícil identificação, principalmente se a expressão algébrica estiver na forma expandida, como por exemplo $f(x)=(x+2)^{2}=x^{2}+4 x+$ 4.

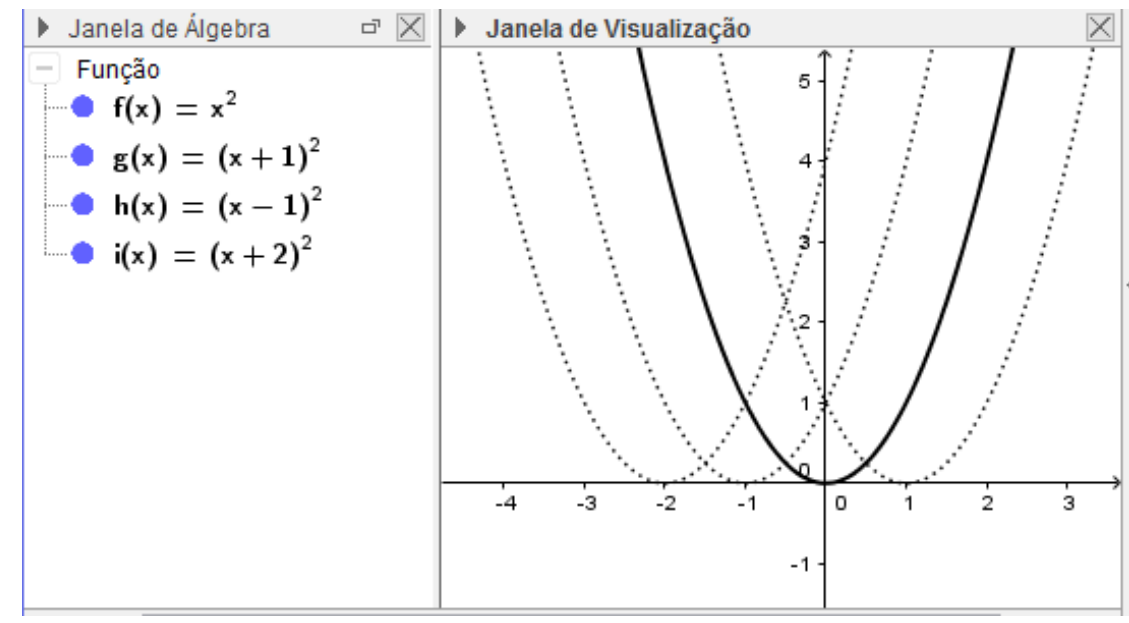

Figura 2: função no GeoGebra

Segundo Duval (2003, 2009), é na capacidade de realiza a conversão de registros que emerge a aprendizagem em matemática, e é isto que pretendemos evidenciar na formação dos professores.

Outra peculiaridade das representações dinâmicas é o conceito de relação funcional entre elementos geométricos, implícito nos diferentes procedimentos de construção disponíveis na barra de ferramentas (GRAVINA, 2001): os elementos iniciais da construção são as variáveis independentes, os elementos finais são as variáveis dependente, e a 'lei' da função é dada pelo procedimento intermediário (com mais ou menos passos de construção automatizados). Por exemplo, na ferramenta Reta Perpendicular do GeoGebra, os elementos iniciais são uma reta $\mathrm{r}$ e um ponto $\mathrm{P}$, e é a partir deles que se obtém o elemento resultante 'reta perpendicular à $r$, passando pelo ponto P'.



Figura 3: relação de dependência

Ou seja, os elementos que aparecem de fato são os elementos iniciais (reta $\mathrm{r}$ e ponto $\mathrm{P}$ ) e o elemento final (reta perpendicular), e todo o processo de obtenção (que é automatizado pelo software) está implícito na representação. Apesar disso, a 
compreensão deste processo implícito é de fundamental importância para a compreensão do processo de produção matemática envolvida.

Outra peculiaridade do GeoGebra (como também em outros softwares similares) tem-se o recurso de 'estabilidade da figura': feita uma construção, a manipulação dos elementos geométricos que dão início à construção transformam o desenho que está na tela do computador, mas as relações geométricas impostas à construção, bem como as relações que delas decorrem, se mantém invariantes. Isto está evidenciado em cada uma das três figuras acima. $\mathrm{Na}$ figura 1 , movimentando-se o vértice $\mathrm{C}$ o triângulo sempre será retângulo

Todas estas peculiaridades da representação dinâmica, conferem à ela uma distinção dos registros previamente apresentados (língua natural, geométrico, gráfico, sistemas de numeração). Para evidenciar estas peculiaridades, o mesmo será denominado de registro de representação dinâmica.

E quando se fala na formação de professores de matemática para o uso de tecnologias, o registro de representação dinâmica possui papel importante, dadas as peculiaridades apresentadas, bem como todas as possibilidades de transformação de registros (tratamento e conversão) envolvidos. E os softwares de MD possuem este potencial diferenciado, que passaremos a chamar de Potencial dos Registros Dinâmicos de Representação Semiótica (PRDRS). E o processo de formação de professores que estamos propondo, se caracteriza por colocar em evidência a exploração do PRDRS por parte dos professores em formação.

\section{Gênese instrumental pessoal e profissional}

A teoria desenvolvida por Rabardel (1995), referida como Abordagem Instrumental, trata do entendimento das ações e reações de um sujeito que interage com um artefato. $\mathrm{O}$ artefato é um dispositivo material ou simbólico ao qual são associados esquemas de utilização. Nesta teoria, é introduzido o conceito de instrumento, de uma forma bastante precisa: a apropriação de um artefato, depende do desenvolvimento de esquemas de utilização é o binômio 'artefato + esquemas-de-utilização' que constitui o instrumento.

Rabardel (1995), considerando as interações do sujeito com o artefato na realização de uma atividade, classifica os esquemas de utilização em duas categorias:

- os esquemas de uso: são aqueles que estão presentes em ações específicas e diretamente relacionadas com o artefato, e que respondem pelas tarefas secundárias, a serem executadas, como parte de uma tarefa maior.

- os esquemas de ação instrumentada: são aqueles que englobam esquemas de uso, em uma totalidade, e que respondem pela realização da tarefa primordial - a tarefa maior. São esquemas complexos, associados a uma significação global das tarefas que realizam a tarefa maior.

Para ilustrar os conceitos elencados acima, pode se considerar um exemplo de Rabardel (1995, p. 92): o artefato é um carro, o sujeito é o motorista e o esquema de ação instrumentada é "realizar uma ultrapassagem". O sujeito precisa coordenar uma série de esquemas de uso (elementares), e é esta coordenação que vai constituir o esquema de ação instrumentada (complexo) que garante a realização da ultrapassagem. Dentre os esquemas de uso que participam do esquema de ação instrumentada tem-se, por exemplo: olhar no espelho retrovisor, ligar o pisca-pisca ou seta, acelerar o carro, girar o volante. 
A ultrapassagem é uma ação instrumentada - para realizar a ultrapassagem, não basta o motorista ter domínio dos esquemas de uso listados; é necessário o domínio de um esquema formado por uma totalidade articulada e simultânea de esquemas de uso - este é o esquema de ação instrumentada.

No estudo, o artefato é o GeoGebra, e se espera que os professores associem esquemas de uso e esquemas de ação instrumentada ao artefato, transformando-o assim, em instrumento. Este processo, da passagem de artefato para instrumento que Rabardel (1995) denomina de Gênese Instrumental.

A partir da literatura, observamos que muitas das vezes os cursos de formação de professores para o uso de tecnologia, possuem enfoque no desenvolvimento de esquemas de uso. Ou seja, a formação está centrada em 'ensinar ao professor como o software funciona'. No entanto, a formação deveria ser centrada em desenvolvimento de esquemas de ação instrumentada, para capacitar o professor na realização de tarefas com o artefato. Este processo de capacitar o professor a realizara tarefas com o software, é o processo de Gênese Instrumental pessoal.

A literatura amplia esta expressão para Gênese Instrumental profissional principalmente quando se trata da incorporação do software na prática docente, com a elaboração de planos de aula, por exemplo. A Gênese Instrumental profissional envolve um processo de autonomia de uso do software pelo professor, não só nas questões de uso do software, mas principalmente no planejamento de atividades didáticas pertinentes e que contribuam significativamente no processo de aprendizagem dos alunos. No processo de Gênese Instrumental, o que se espera é que o professor seja capaz de explorar o potencial semiótico (STORMOWSKI; GRAVINA; LIMA, 2013), em que o software é utilizado como mediador no processo de aprendizagem.

De modo geral, as gêneses pessoal e profissional são processos que ocorrem simultaneamente, mas a primeira é condição para a segunda, já que não é possível, por exemplo, criar planos de aula envolvendo um software, sem que saiba utilizar o software no contexto pessoal, pelo menos em algum nível básico.

No nosso estudo, que envolve as primeiras etapas da formação de professores para o uso de tecnologias, o foco têm sido a Gênese Instrumental pessoal, que poderá ser observada em algumas referências na seção seguinte.

\section{Implicações para a formação de professores de matemática em EAD}

Os aportes teóricos apresentados, bem como demais referências no processo de formação de professores, conduziram o nosso estudo para elencar alguns itens importantes quando se trata da formação de professores de matemática para o uso de tecnologia, através de cursos na modalidade EAD:

- observar as características da formação em EAD, e produzir material e recursos que possam garantir a eficiência da formação nesta modalidade;

- explorar o PRDRS, de modo a capacitar o professor na articulação do conhecimento matemático através dos softwares de $\mathrm{MD}$, conhecimento este que nem sempre é muito evidente;

- formação com foco em atividades que desenvolvam esquemas de ação instrumentada, que mesmo sendo mais complexos, articulam diferentes esquemas de uso, e além disso fornecem subsídios importantes para que o professor desenvolva as suas próprias atividades didáticas. 
O primeiro item não é objeto deste artigo, mas resultou na produção de material rico em situações didáticas, e com suporte adequado para a formação em EAD. Um dos recursos desenvolvidos foi a MultiTela GD (STORMOWSKI; GRAVINA; LIMA, 2014), que além de articular diferentes registros de representação semiótica, contribuiu sensivelmente para o processo de capacitação em EAD.

Os outros dois itens passam a ser ilustrados em dois exemplos de atividades, dentre outras que foram desenvolvidas para o curso.

No primeiro exemplo, ilustrado na figura 4, os professores são explorados a explorar o dinamismo dos triângulos, e identificar as propriedades subjacentes (as retas pontilhadas não ficam visíveis, as circunferências sim).



Figura 4: triângulos dinâmicos

As figuras apresentam propriedades implícitas que nem sempre são fáceis de identificar, e precisam de articulação com conhecimento matemática pertinente. É movimentando os vértices do triângulo (tratamento no registro geométrico) que é possível identificar que a figura verde e azul, embora pareçam ser semelhantes, possuem características muito distintas.

A figura azul começa pela construção da circunferência, e depois basta marcar três pontos sobre ela para obter o triângulo FGH. A figura verde começa pelo triângulo $\mathrm{ABC}$, e para obter a circunferência circunscrita é preciso articular conhecimento de propriedades da mediatriz para obter o circuncentro (centro da circunferência circunscrita ao triângulo). Esta exploração não é evidente para a maioria dos professores que participaram do curso, e precisa que explorem o PRDRS para sua obtenção.

$\mathrm{Na}$ figura vermelha, o processo é mais complexo ainda, pois além de envolver propriedades das bissetrizes par obter o incentro, necessita que se se envolva as propriedades de retas tangentes á circunferência, caso contrária, a circunferência não permanecerá inscrita quando os vértices forem movimentados.

É possível observar que este tipo de atividade não faz uso de orientações para utilizar um ou outro recurso do software, mas a efetivação da tarefa, faz que diversos esquemas de uso sejam mobilizados como consequência.

Um outro exemplo é ilustrado pela figura 5. Nela os participantes do curso são convidados a criar inicialmente uma situação geométrica, em que seja possível identificar relações de dependência entre os elementos geométricas. Na figura, movimentando o ponto vermelho o mesmo percorre a circunferência, e o retângulo roxo vai modificando medidas de lados, apresentado variação na área que depende do lado indicado. Esta relação de dependência é transferida para as coordenadas de um ponto, que percorre o gráfico à direita, à medida que movimentamos o ponto vermelho. 
Este é um exemplo de conversão de registro (do geométrico para o gráfico) em que não se utiliza a expressão algébrica da função. A expressão algébrica poderá ser obtida posteriormente, mas não é condição para a obtenção do gráfico. Para a obtenção do gráfico, os professores em formação costumam confundir o formato do gráfico obtido com uma parábola, o que é um equívoco pois o gráfico não é simétrico.

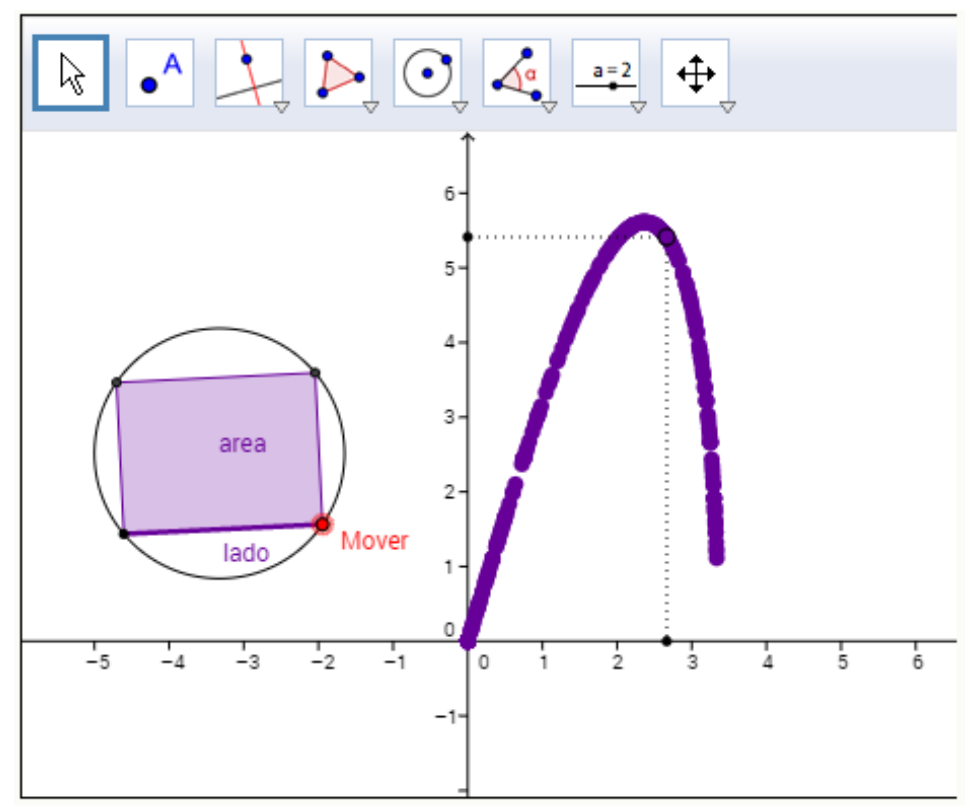

Figura 5: relação entre funções e geometria

Esta é uma situação pouca exploradas nas escolas, onde os livros didáticos costumam enfatizar o processo de obtenção do gráfico a partir da expressão algébrica, e não o contrário. Algo que também se observa pouco nas escolas é a relação entre geometria e funções, evidenciadas nesta atividade.

Estas são algumas tarefas, dentre diversas outras, que efetivam um plano de formação de professores de matemática para o uso de tecnologias, em que os mesmos explorem o PRDRS.

A partir do estudo ainda em desenvolvimento, podem ser observados diversas contribuições deste tipo de enfoque, principalmente na capacitação do professor, que o leve na direção de desenvolver a autonomia no uso do software, elaborando situações didáticas que potencializem o processo de aprendizagem de seus respectivos alunos

\section{Considerações finais}

As contribuições dos registros de representação semiótica (DUVAL, 2009) e a ampliação para os registros dinâmicos de representação dos softwares de MD, bem como a abordagem instrumental (RABARDEL, 1995), contribuíram efetivamente para a obtenção de itens chave na elaboração de cursos de formação de professores de matemática para o uso de tecnologia.

Em uma análise inicial de dados obtidos, observa-se que um processo rápido de apropriação do software, mesmo para professores de matemática que nunca tinham tido contato com o software GeoGebra. No entanto, é um estudo ainda em desenvolvimento e que se espera possa apontar outros indícios de fatores que contribuam com este tipo de formação. 
As situações apresentadas envolveram o software GeoGebra, mas estratégias envolvidas podem ser adaptadas para outros recursos digitais que contribuem para a aprendizagem matemática. Esta, inclusive, é uma das continuidades do estudo em andamento.

O que se espera, é que a formação que tenha as características apresentadas, possa contribuir para que se mude o cenário de uso de tecnologias no contexto escolar, apresentado na introdução, e que as tecnologias passem ser utilizadas efetivamente, com contribuições significativas, no processo de aprendizagem em matemática.

\section{Referências}

ARTIGUE, M. The future of teaching and learning mathematics with digital technologies. In: HOYLES, C.; LAGRANGE, J.-B. (Org.). Mathematics Education and Technology-Rethinking the Terrain. [S.1.]: Springer US, 2010. p.463475. (New ICMI Study Series, vol. 13).

BARBOSA, A. (Org.). Pesquisa sobre o uso das tecnologia de informação e comunicação no Brasil: tic educação 2011. São Paulo: Comitê Gestor da Internet no Brasil, 2012.

BARBOSA, A. (Org.). Pesquisa sobre o uso das tecnologias de informação e comunicação nas escolas brasileiras: tic educação 2013. São Paulo: Comitê Gestor da Internet no Brasil, 2014.

DUVAL, R. Registros de representações semióticas e funcionamento cognitivo da compreensão em matemática. In: Aprendizagem em matemática: registros de representação semiótica. Campinas: Papirus, 2003. p. 11-34.

DUVAL, R. Semiósis e Pensamento Humano: registros semióticos e aprendizagens intelectuais. São Paulo: Livraria da Física, 2009.

ERNEST, P. A semiotic perspective of mathematical activity. Educational Studies in Mathematics, Dordrecht, vol. 61, 2006.

JOUBERT, M. Using digital technologies in mathematics teaching: developing an understanding of the landscape using three "grand challenge" themes. Educational Studies in Mathematics, vol. 82, n. 3, p. 341-359, mar. 2013.

RABARDEL, P. Les hommes et les technologies: aprproche cognitive des instruments contemporains. Paris: Armand Colin, 1995.

STORMOWSKI, V.; GRAVINA, M. A.; LIMA, J. V. Tecnologia na aula de matemática: a importância do potencial semiótico. RENOTE - Revista Novas Tecnologias na Educação, Porto Alegre, vol.11, n. 3, Dezembro 2013.

GRAVINA, M. A. Os ambientes de geometria dinâmica e o pensamento hipotético-dedutivo. 2001. Tese (Doutorado em Informática na Educação) UFRGS, Porto Alegre.

STORMOWSKI, V.; GRAVINA, M. A.; LIMA, J. V. MultiTela GD: um recurso para a formação de professores de matemática em ead. RENOTE - Revista Novas Tecnologias na Educação, Porto Alegre, vol. 12, n.2, Dezembro 2014. 